INVESTIGACIONES

\title{
Sentido de Futuro en Estudiantes Secundarios: Paradojas de Equidad y Calidad desde su Experiencia Escolar
}

\author{
Sense of Future in High School Students: Paradoxes of Quality and Equity from the \\ School Experience \\ Sentido de Futuro para Estudantes do Ensino Médio: Paradoxos da Equidade e da \\ Qualidade desde sua Experiências Escolares
}

\begin{abstract}
Walter Molina Ch.
Universidad de Magallanes, Departamento de Ciencias Sociales. Correo electrónico: walter.molina@umag.cl. Telf.: (61) 2207135
\end{abstract}

\begin{abstract}
RESUMEN
El presente artículo aborda el sentido de futuro de estudiantes secundarios que asisten a liceos municipales en Chile. En torno a dichas expectativas de futuro, emergen algunas paradojas de calidad y equidad que se manifiestan en sus discursos sobre los procesos de escolarización secundaria. La relevancia de aproximarnos a esta paradoja a través del análisis del sentido de futuro de los estudiantes, responde a la necesidad de abordar la dimensión subjetiva y su incidencia en las posibilidades de mejoramiento de la educación secundaria. En esta perspectiva, se integra en el análisis tanto dimensiones estructurales como subjetivas de los procesos de escolarización en Chile. Al respecto, Corica (2012) sostiene que no sólo los cambios ocurridos en el mercado del trabajo y en el sistema educativo operan en la visión de futuro de los jóvenes; sino que existen otros factores sociales y representacionales que inciden en la configuración de dichas expectativas.
\end{abstract}

Palabras clave: sentido de futuro, educación secundaria, equidad, calidad.

\begin{abstract}
This article discusses the sense of future in high school students attending municipal schools in Chile. Around these expectations of future emerge some paradoxes of quality and equity which are manifested in their speeches on municipal education. Therefore, studying these paradoxes allows an understanding of the subjective dimension in secondary schooling processes and their possibilities of improvement. From this perspective, we are interested in integrating to the analysis both, the structural and subjective, dimensions of the processes of secondary schooling in Chile. In this regard, Corica (2012) argues that not only the changes in the labor market and in the educational system operate in the vision of future of young people, but there are also other social and representational factors that strongly influence the configuration of such expectations.
\end{abstract}

Key words: sense of future, high school, quality, equity.

\section{RESUMO}

Aborda-se o sentido de futuro de estudantes que frequentam escolas de Ensino Secundário municipais no Chile; em torno das expectativas de futuro deles emergem alguns paradoxos de qualidade e equidade manifestados em seus discursos sobre os processos de escolarização secundária. A relevância de aproximação de esses paradoxos por meio da análise de sentido de futuro dos estudantes enquadra a necessidade de abordar a dimensão subjetiva e sua incidência nas possibilidades de melhoramento da escolarização secundária. Com esta perspectiva, integram-se à análise tanto dimensões estruturais como subjetivas dos processos de escolarização no Chile. A este respeito, Corica (2012) sustenta que não apenas as transformações ocorridas no mercado de trabalho e no sistema educacional afetam a visão de futuro dos jovens; há outros fatores sociais e representacionais que incidem na configuração de essas expectativas.

Palavras chave: sentido de futuro, educação secundária, equidade, qualidade.

* Este artículo forma parte del "Programa Postdoctoral de Investigación en Ciencias Sociales, Niñez y Juventud" (Centro de Estudios Avanzados en Niñez y Juventud (CINDE)-Universidad de Manizales-Colombia; Pontificia Universidad Católica de São Paulo-Brasil; Universidad Católica Silva Henríquez-Chile y el Consejo Latinoamericano de Ciencias Sociales (CLACSO)-Argentina). 


\section{INTRODUCCIÓN}

La sociedad chilena está experimentado profundas transformaciones en las diversas esferas de lo social. Estos cambios se han evidenciado especialmente en el campo educativo, los que, analizados en su conjunto, han producido nuevas formas de configuración del lazo social complejizando la tradicional relación que los sujetos establecían con algunos ámbitos claves de la vida social: trabajo, familia y conocimiento. A juicio de Araujo \& Martuccelli (2012) estos cambios han reconfigurado los desafíos comunes o pruebas sociológicas ${ }^{1}$ a los que están sometidos los sujetos sociales (y educativos) en la sociedad chilena contemporánea. En consecuencia, junto con modificar la estructura de nuestra sociedad, están generando profundas transformaciones a nivel de la subjetividad, emergiendo nuevos modos de articulación entre individuos, instituciones educativas y sociedad.

En este contexto, se ha producido una modificación radical en la estructura y fundamentos del sistema educativo chileno. Una manifestación de estos cambios es el explosivo crecimiento de las tasas de matrícula en todos los niveles educativos (básico, medio y superior). A modo de ejemplo, la enseñanza secundaria en los años 60 alcanzaba un $14 \%$ de cobertura para su respectiva cohorte de estudiantes. En los inicios de los años noventa el sistema escolar secundario había logrado una cobertura de $75 \%$, constituyéndose definitivamente en un sistema de acceso masivo con una cobertura cercana a $80,5 \%$ (MINEDUC, 2009, 2010). Es decir, ocho de cada diez jóvenes se encuentra inserto en alguna modalidad de educación secundaria. Sin embargo, de acuerdo a diversos estudios, la amplia cobertura descrita no estaría asociada a los niveles de calidad deseados por el sistema educativo, lo cual ha generando un conjunto de inequidades e ineficiencias del sistema y una pérdida progresiva de su eficacia externa como mecanismo de movilidad social ascendente (OCDE, 2010; MINEDUC, 2010).

Cabe señalar que, en este marco de expansión sistemática de la matrícula de educación media, y desde inicios de los años ochenta, las políticas educativas chilenas han transitado desde un sistema educacional estatal, en el cual el Estado cumplió un activo rol en la producción, financiamiento y asignación/distribución de los servicios educativos; pasando a la conformación de un sistema educativo basado en la asignación de recursos a través de mecanismos de mercado. Para Bellei (2012), la fuerza motriz que logró la expansión del sistema educacional chileno fue reemplazada por la libre interacción de la oferta y la demanda, conformándose propiamente un mercado escolar.

Las deficiencias del sistema educativo se han manifestado en una pérdida progresiva de la capacidad efectiva de agregar valor a la educación en términos de resultados de aprendizaje de competencias consideradas básicas para una adecuada inserción social y laboral de los egresados. Al respecto, recientemente la Unesco (2011) ha señalado que, a pesar que en muchos países aumentó la incorporación de jóvenes al nivel secundario, los niveles de calidad y su relevancia/pertinencia sociocultural aún representan grandes desafíos para los sistemas educacionales de los países latinoamericanos, incluido Chile.

Es una noción teórica que busca articular los problemas personales con las estructuras sociales que los crean o amplifican. Su objetivo es lograr caracterizar precisamente los desafíos a los que son sometidos los actores y las maneras en que los individuos superan o intentan superar las pruebas. La noción permite articular lo individual y lo colectivo en la explicación (imaginación) sociológica (Martuccelli, D. \& de Singly, 2012). 
En un análisis comparativo Bellei (2012) sostiene que mientras las tasas de graduación de la educación secundaria en países de mayor desarrollo son iguales o superiores a $90 \%$ para el grupo de edad de referencia (14-17 años), en América Latina se ubican entre $40 \%$ y $60 \%$, sin superar el $70 \%$ entre los países con mejores indicadores educativos. Más aún, si se compara en términos de logros de aprendizaje alcanzados por los alumnos latinoamericanos, la situación sigue siendo crítica. De acuerdo con la prueba PISA (OCDE, 2009), más de un tercio de los alumnos chilenos y mexicanos de 15 años está bajo el nivel de lectura considerado básico; y en países como Perú, Argentina, Colombia y Brasil, al menos la mitad de los estudiantes de 15 años no ha adquirido dicho nivel de desempeño. Frente a este negativo diagnóstico, son evidentes los desafíos que tiene la educación secundaria en términos de sus niveles de calidad y de superación de inequidades en el acceso, progreso, retención y conclusión de este ciclo por parte de los estudiantes de los estratos socioeconómicos más vulnerables de la sociedad.

Desde una perspectiva global es importante considerar que la escuela secundaria, tanto en Chile como en América Latina, emerge históricamente tensionada por objetivos y funciones sociales contradictorias. Por una parte, se le asigna como objetivo favorecer la incorporación e integración de todos los estudiantes a sus ciclos educativos. Y, por otro lado, continuar con su mandato fundacional de seleccionar académica y culturalmente a "los más aptos" (Ferreira, 2006: 2). Es decir, y tal como dice Bellei (2012: 217) la escuela secundaria estaría tensionada por intentar compatibilizar principios que se contraponen entre sí; ser meritocrática y compensatoria a la vez; por una parte, constituir un ciclo educativo terminal y, por otra parte, preparatoria, integradora y selectiva, es decir, estar abierta a los intereses de los jóvenes y de la sociedad simultáneamente. Y, en este mismo contexto de demandas contradictorias, se encuentra la tensión esencial y no resuelta de la educación secundaria: ¿Preparar para el trabajo y/o la continuación de estudios superiores? Frente a esta disyuntiva, se intenta abordar la complejidad de la relación sujeto/escuela. Por ello, desde nuestra perspectiva de análisis, resulta relevante indagar en el sentido de futuro que construyen desde la propia experiencia escolar las niñas, niños y jóvenes que habitan cotidianamente el espacio escolar de la educación secundaria pública en Chile.

\section{PROCESOS DE ESCOLARIZACIÓN SECUNDARIA EN CHILE Y DESAFIOS DE EQUIDAD Y CALIDAD}

En América Latina en general y Chile en particular, en las últimas décadas se ha logrado expandir significativamente la matrícula de la educación primaria y secundaria, es decir, se extendió la cobertura de estos servicios educativos a sectores de la población que tradicionalmente estaban al margen de dicha oferta educativa. En consecuencia, el desarrollo educativo en Chile y en América Latina, tiende a la masificación de la escolarización secundaria en un contexto marcado por fuertes desigualdades socioeconómicas. A modo de ejemplo, Tenti (2007) destaca que la masificación de la escolaridad media ha operado junto a un profundo proceso de segmentación de la oferta educativa asociándose

PISA: Programme International Student Assessment. Es una prueba estandarizada que se aplica en los países miembros de la Organización para la Cooperación y el Desarrollo Económico (OCDE). 
su calidad según el grupo socioeducativo al que pertenece el estudiante. ${ }^{3}$ Lo anterior se manifiesta en acceso altamente segmentado a una escolarización de "élite"; al mismo tiempo, los sectores de menores recursos acceden a una educación pública (municipal para el caso de Chile) que ha experimentado un continuo proceso de no solo pérdida de calidad educativa, sino además de eficacia externa, es decir, como mecanismo de movilidad social ascendente alejando la posibilidad de construir trayectorias sociales más integradoras. Para Pereira Leaõ (2012) se trataría de una forma de desigualdad o más precisamente de un proceso de inserción a medias (Martins, 1997). Este autor sostiene, para el caso brasileño, que la mayoría de los estudiantes socializa en el contexto de una experiencia de inserción escolar frágil que no les responde como un amplio proceso de formación humana y que no visualizan como capaz de promover su inserción social y/o profesional digna en el futuro. Al respecto, Pereira Leaõ (2012: 126) señala que varios estudios constataron que la expansión de la escolaridad en Brasil representó más bien un cuadro de masificación de la educación, de ampliación del acceso, sin que ello significara un proceso real de democratización, en términos de equidad y calidad educativa.

Esta situación es preocupante y contradictoria con el amplio consenso existente entre diversos autores y organismos internacionales (Macedo \& Katzkowicz, 2002; Tedesco \& Lopez, 2002; Rodríguez, 2008; SITEAL, 2008; CEPAL, 2000, 2008, 2011; UNESCO, 2011. UNESCO, 2012) en relación a la importancia estratégica de la educación secundaria tanto para los procesos de desarrollo social; como para las trayectorias socioeducativas de los sujetos que egresan la escuela secundaria en nuestro continente. En este sentido, la CEPAL (2000) sostiene que tener al menos 12 años de escolaridad es considerado como capital educativo mínimo necesario para alcanzar un nivel satisfactorio de bienestar. Asimismo, se considera que completar este nivel de escolaridad genera una alta probabilidad $(80 \%)$ de conseguir una ocupación e ingresos para lograr un nivel de vida adecuado. En este contexto, la educación secundaria constituiría un factor protector clave para conseguir oportunidades de trabajo incrementando la posibilidad de superar la línea de pobreza (UNESCO, 2012).

En Chile, durante las dos últimas décadas se ha implementado un conjunto de políticas educativas dirigidas a renovar y fortalecer el nivel educativo secundario: aumento de cobertura, cambios curriculares, mayor infraestructura, regulación de la profesión docente, entre otras medidas relevantes. Estas reformas de primera generación (Eyzaguirre, 2010) estuvieron dirigidas a producir mayor equidad en el acceso a los servicios educativos, focalizando sus objetivos en mejorar, tanto la disponibilidad de recursos educativos como fortalecer los procesos de gestión educativa. No obstante, diversos autores (OCDE, 2004, Bellei \& González, 2010; Eyzaguirre, 2010; García-Huidobro, 2010) sostienen que pese a las significativas mejoras introducidas, este nivel educativo no ha logrado superar sus problemas estructurales y aún persisten importantes desafíos que cumplir en materias tales como: segregación escolar, disminuir la deserción escolar, mejorar los niveles de aprendizajes, aumentar los resultados en las pruebas estandarizadas aplicadas a nivel internacional (PISA, 2009) y eliminar las inequidades del sistema educativo, entre otros

La escolarización secundaria se ha hecho jurídicamente obligatoria. "También se observa un crecimiento significativo en la escolarización de los adolescentes. La asistencia escolar en la franja de edad de 13 a 19 años en el Brasil, pasa del 64,6\% (1990) al 77,5\%(2001), en México la tasa va del 62,7\% (1992) al 68,9\% (2002) y en Argentina sube del 68,8\% (1990) al 83,2\% (2002)" (Tenti, 2007: 58). 
múltiples desafíos pendientes. En virtud de lo anterior, el desafío más urgente, consiste en impulsar reformas educativas de segunda generación, es decir, aquellas dirigidas a implementar cambios en las reglas básicas del sistema educativo que generen una educación pública de igual calidad para toda la población escolar que la demande y a la cual acceda obligatoria y gratuitamente.

Esta problemática y el requerimiento de equidad se ha evidenciado en las sucesivas demandas y movilizaciones de los estudiantes secundarios entre los años 2006 y 2012. En ellas los diversos actores socioeducativos (estudiantes, padres, profesores) han construido un doble consenso en torno a los problemas del sistema educativo chileno; en primer lugar, se plantea que dichos problemas tienen un origen estructural en tanto han sido consecuencia del modelo educativo neoliberal implementado en Chile en los últimos 40 años, generando desigualdad e inequidades en el acceso a la oferta educativa; así como deficientes resultados de aprendizajes al finalizar los respectivos ciclos educativos. Por otra parte, se plantea que la motivación centrada en el lucro entre los oferentes privados de servicios educativos ha acelerado el fortalecimiento del sistema privado.

\section{EDUCACIÓN SECUNDARIA EN CHILE: OBJETIVOS, COBERTURA Y POLÍTICAS EDUCATIVAS DE EQUIDAD Y CALIDAD}

\subsection{SURGIMIENTO DE LA EDUCACIÓN SECUNDARIA EN CHILE}

La enseñanza secundaria, considerada como un tramo escolar con objetivos, finalidades y contenidos propios, fue una creación republicana de mediados del siglo XIX en Chile. Este nivel educativo fue diseñado paralelamente a la independencia de nuestro país. Su primera manifestación habría sido en el plan de estudios de $1832,{ }^{4}$ sin embargo, a partir de 1843 se implementó de manera definitiva a través del plan de estudios humanista, inspirado por Domeyko (Cruz, 2002: 20) y el cual se convierte en la matriz analítica de debates sobre la orientación y los fines de la educación en general y de la educación secundaria en particular. Al respecto, Bellei (1995) sostiene que gran parte de lo que está hoy en las discusiones sobre la "crisis de la educación media", "sus reformas" y "necesarias modernizaciones educativas"; son reediciones actualizadas de las preocupaciones, debates y argumentos de nuestros antecesores, a saber: la contribución de este nivel al desarrollo económico del país, el carácter de los conocimientos a transmitir en el nivel medio (conocimientos generales o especializados) y sus desafíos de democratización y expansión de la ciudadanía hacia los cuales este nivel educativo debe orientarse (Bellei, 1995: 12).

En síntesis, este nivel educativo ofrece, casi desde sus orígenes, una formación general común y formaciones diferenciadas (humanístico-científica, técnico-profesional

Plan Propuesto por Ventura Marín, Manuel Montt y Juan Godoy, comprendía seis años de estudios comunes en el curso de humanidades. En cada uno de ellos había una clase principal, una subalterna y una accesoria. Durante los primeros cuatro años se estudiaba latín como clase principal; geografía como clase subalterna y elementos de aritmética como clase accesoria. En los dos años últimos años, se estudiaba filosofía mental como clase principal (quinto año) y finalmente filosofía moral y derecho natural (sexto año) como clase principal; Griego como clase subalterna y Francés o Inglés como clase accesoria. Al término de estos seis años de estudio se ubicaba un curso de leyes, uno de medicina, uno de matemática y otro teológico, es decir, la formación propiamente científica o profesionalizante (Cruz, 2002: 68). 
y artística). Por otra parte, se ha constituido históricamente en un puente que busca atender a la población escolar que ha finalizado el nivel de enseñanza básica y tiene por finalidad procurar que cada alumno, mediante el proceso educativo sistemático, expanda y profundice su formación general y desarrolle los conocimientos y competencias que le permitan ejercer una ciudadanía activa e integrarse a la sociedad. En un análisis más global, la UNESCO (2011) sostiene que este nivel educativo representa una etapa crítica del sistema educativo ya que, además de vincular la educación inicial o primaria con la educación superior, tiende puentes en el sistema escolar y el mercado del trabajo. Por ello, las actuales políticas educativas de los países en los cuales se ha masificado la educación secundaria, deben responder urgentemente a los desafíos de generar mayores niveles calidad, equidad (tanto en el acceso como en el egreso oportuno), pertinencia sociocultural de los procesos educativos y, sobre todo, lograr relevancia social de los aprendizajes, conocimientos y competencias adquiridas al finalizar este importante ciclo educativo.

Como una forma de responder a las crecientes demandas de los actores socioeducativos por mejorar los niveles de calidad y equidad en la educación escolar chilena, recientemente se promulgó la Ley 20.501 (2011) de Calidad y Equidad de la Educación. En dicho cuerpo legal se consagra, fundamentalmente, un conjunto de normas administrativas y de gestión tendientes orientadas a lograr mejores resultados educativos; así como ajustar los recursos financieros a las crecientes necesidades asociadas a proveer educación de calidad tanto en la educación básica como en la educación media. Cabe señalar que dicha ley solo se centra aspectos y procesos técnico administrativos de la gestión escolar, tanto en escuelas básicas como secundarias, y llama la atención que no quedan definidos los conceptos de calidad y equidad de la educación; y mucho menos sus indicadores de logro y los estándares hacia los cuales las políticas educativas buscan llegar.

\subsection{EVOLUCIÓN DE LA MATRÍCULA DE LA EDUCACIÓN SECUNDARIA PÚBLICA EN CHILE: ASPECTOS RELEVANTES SU PROCESO DE MASIFICACIÓN Y OBLIGATORIEDAD}

Como una forma de contextualizar la posterior presentación de datos sobre la configuración de subjetividades en torno la noción de futuro postsecundario, y las paradojas tanto de calidad como de equidad que emergen en los discursos de los estudiantes de liceos municipales de distintos estratos en la región metropolitana en Santiago de Chile, se presentan algunos datos generales sobre la evolución de la matrícula en educación media, los que expresan el número bruto de estudiantes que el sistema incorpora en diversos períodos de su desarrollo. Por otra parte, se presenta información general acerca de los niveles de cobertura que esta matrícula representa en referencia al grupo socioeducativo hacia el cual está dirigida esta importante política educativa (14 a 17 años de edad).

En este contexto, cabe señalar que hacia el año 1900 el conjunto del sistema educacional alcanzaba los 114.410 estudiantes matriculados en los tres niveles del sistema, de los cuales la enseñanza media llegaría a representar un 30\%. Como podemos observar, la educación media experimentó un crecimiento significativamente menor que el nivel básico, no llegando a triplicarse durante el período, pese a ser la más promovida por las autoridades educacionales y recibir una asignación de recursos significativamente superior a los otros dos niveles educativos (Cruz, 2002: 119).

En relación al segundo hito en el desarrollo de la enseñanza media en Chile, y que corresponde al período 1935-1988, se puede afirmar que durante este periodo la 
matrícula experimentó un explosivo crecimiento que transformó este nivel educacional de una educación secundaria "de elite" a una educación secundaria "de masas". Al final del período analizado, se logra una matrícula bruta de 735.701 estudiantes secundarios; lo que representa un $60.2 \%$ de cobertura. Sin embargo, estos logros, que tienden hacia la universalización de la educación media, generan nuevos problemas en el sistema educativo: inequidades en el acceso a la educación media, altas tasas de deserción escolar, segmentación de la oferta educativa según los ingresos socioeconómicos de las familias de los estudiantes (escuelas municipales, subvencionadas y pagadas), bajos resultados de aprendizaje, entre otros problemas emergentes.

La evolución de la matrícula de la enseñanza media entre 1990 y el 2002, corresponde a un periodo en el cual se ha consolidado la masividad de este nivel educativo. Los datos que ilustran esta evolución son los siguientes:

Figural. Evolución tasas de matrícula y cobertura de la Enseñanza Media (15-19 años)

\begin{tabular}{|c|c|c|c|c|c|c|}
\hline Año & $\begin{array}{c}\text { MATRICULA } \\
\text { Educación } \\
\text { MediaTotal }\end{array}$ & $\begin{array}{c}\text { Porcentaje } \\
\text { de Grupo } \\
\text { 14-17 años }\end{array}$ & $\begin{array}{c}\text { Matrícula } \\
\text { Humanista- } \\
\text { Científico }\end{array}$ & $\begin{array}{c}\text { Porcentaje } \\
\text { Total }\end{array}$ & $\begin{array}{c}\text { Matrícula } \\
\text { Técnico } \\
\text { Profesional }\end{array}$ & $\begin{array}{c}\text { Porcentaje } \\
\text { del Total }\end{array}$ \\
\hline 1981 & 554.749 & $65 *$ & 392.940 & 70.8 & 161.809 & 29,2 \\
\hline 1990 & 699.455 & 77 & 436.892 & 62.5 & 262.563 & 37,5 \\
\hline 1991 & 699.455 & 77 & 436.992 & 62,5 & 262.563 & 37,5 \\
\hline 1992 & 675.073 & 77 & 410.896 & 60.0 & 264.177 & 40.0 \\
\hline 1993 & 652.815 & 76 & 391.457 & 60.0 & 261.358 & 40.0 \\
\hline 1994 & 664.498 & 76 & 387.272 & 58,3 & 277.226 & 41,7 \\
\hline 1995 & 688.440 & 78 & 388.177 & 56,4 & 291.048 & 43,6 \\
\hline 1996 & 739.316 & 80 & 415.919 & 56,3 & 323.397 & 43,7 \\
\hline 1997 & 753.250 & 83 & 421.132 & 55,9 & 332.118 & 44,1 \\
\hline 1998 & 774.034 & 83 & 424.892 & 54,9 & 349.142 & 45,1 \\
\hline 1999 & 803.832 & 83 & 441.281 & 55,3 & 359.551 & 44,7 \\
\hline 2000 & 822.946 & 84 & 456.246 & 55,4 & 366.700 & 44,6 \\
\hline 2001 & 850.713 & 85 & 476.641 & 58,8 & 376.072 & 44,2 \\
\hline 2002 & 896.470 & 87 & 513.813 & 57,3 & 382.657 & 42,7 \\
\hline
\end{tabular}

Fuente: Cox, C. (2003).

Como se puede observar, en el periodo analizado se produce un sistemático crecimiento de la matrícula y un aumento significativo de la cobertura, la cual pasa desde el $65 \%$ al $87 \%$ respectivamente. Por otra parte, y como una forma de aportar datos de matrícula que ilustran el desempeño de estos indicadores en los últimos años, se presenta la información disponible sobre la evolución de la tasa neta de matrícula en la educación secundaria chilena el periodo 2004-2008: 


\begin{tabular}{|c|c|c|c|c|c|}
\cline { 2 - 6 } \multicolumn{1}{c|}{} & 2004 & 2005 & 2006 & 2007 & 2008 \\
\hline Matricula Educación Media & $75,8 \%$ & $79,7 \%$ & $81,1 \%$ & $80,8 \%$ & $80,5 \%$ \\
\hline
\end{tabular}

Fuente: MINEDUC (2009) Indicadores de la Educación en Chile 2007-2008.

Como se puede observar, la evolución de la tasa neta de matrícula de la enseñanza secundaria en Chile se consolida en torno al $80 \%$ de cobertura, confirmando su carácter masivo y obligatorio, aunque no gratuito, como uno de los principales atributos del sistema educacional chileno en los inicios del Siglo XXI. Sin embargo, es necesario destacar que esa amplia cobertura convive con una tasa de deserción escolar que se ha mantenido en torno al $7 \%$ anual, lo que constituye una de las principales barreras que impiden universalizar este nivel educativo. Lo anterior es una de las múltiples evidencias para saber que esta explosiva expansión de la matrícula de educación media en Chile, se produce en un escenario socioeducativo marcado por graves problemas de calidad y equidad.

\subsection{RESULTADOS DE LA EDUCACIÓN MEDIA Y SU RELACIÓN PARADOJAL ENTRE CALIDAD Y EQUIDAD}

Las consecuencias de los procesos de expansión de la enseñanza secundaria, tanto en Chile como en América Latina; se relacionan con fenómenos más globales y complejos que experimentan las sociedades modernas y particularmente las sociedades latinoamericanas. Para algunos autores (Flecha y Tortajada, 2004), la masificación de la escolarización con bajos niveles de calidad es uno de los síntomas más evidentes de la "crisis de la escuela moderna". Surgen, de este modo, nuevos problemas educativos: déficit de calidad, alta heterogeneidad del alumnado, rigidez curricular, inequidades en el acceso de diversos segmentos de la población a los servicios educativos, devaluación de las certificaciones adquiridas en las diversas modalidades de enseñanza, problemas de convivencia escolar, entre otros problemas relevantes de la educación en pleno Siglo XXI.

Las grandes diferencias que se producen entre los logros de aprendizaje en la pública (municipal y particular subvencionada) y la particular pagada es otro de los antecedentes asociados a la perdida de relevancia social de la escolarización secundaria. Estos desiguales logros hacen que académicamente sea muy difícil el acceso a la educación superior y/o la inserción laboral para los jóvenes de familias de bajos ingresos y que estudian en liceos municipales. Sin embargo, estos logros diferenciales eran muy difíciles de medir en ausencia de una evaluación externa al sistema secundario que no fuera, por supuesto, la PSU, cuyos puntajes no están estandarizados en base al promedio obtenido por los estudiantes. Al respecto, se destaca el estudio de Arzola et al. (1993) en el que se realiza una evaluación global de la eficiencia externa de la enseñanza media. Este estudio se focaliza en la inserción de sus egresados considerando las trayectorias para las cuales fueron específicamente preparados. Los datos son los siguientes: 
Fig. 2. Inserción Educacional de los egresados de Enseñanza media (en \%) Total Nacional 1990

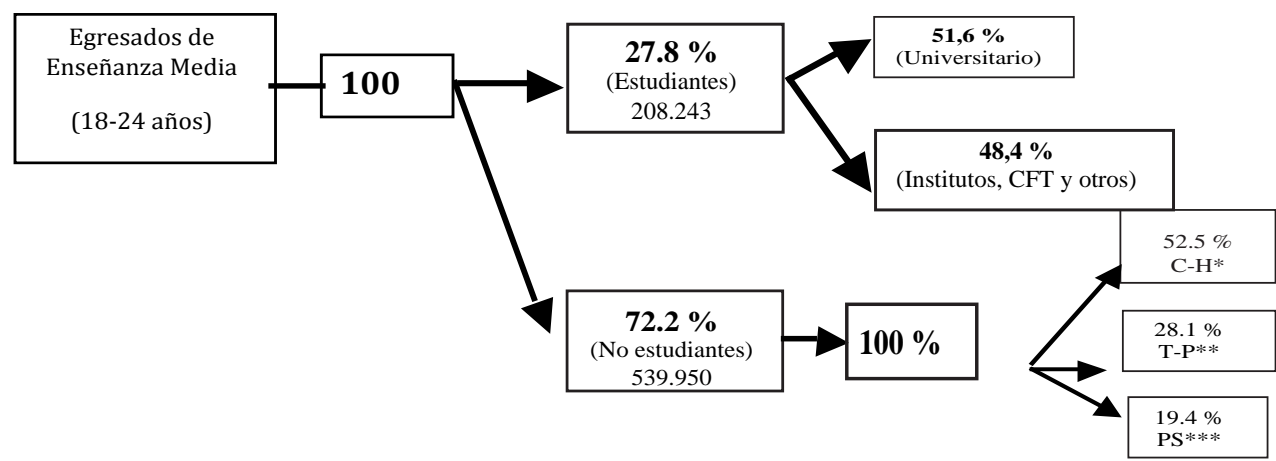

Fuente: Arzola (1993: 19). * Egresados de la modalidad científico- humanista. **Egresados de enseñanza TécnicoProfesional **Egresados con educación Postsecundaria, pero que al momento de la encuesta no eran estudiantes.

En este cuadro se observa un alto porcentaje de egresados que no se han incorporado a la Educación Superior una vez que han completado sus estudios secundarios $(72,2 \%)$ y que no necesariamente se han insertado al mercado laboral; en segundo lugar, se encuentran los egresados de la enseñanza media técnico profesional (Arzola, 1993: 18). Por su parte, de los egresados que prosiguen estudios superiores (208.243) un 51.6\% se encontraba cursando estudios propiamente universitarios y un $48.4 \%$, realizaba estudios no universitarios (Institutos Profesionales y Centros de Formación Técnica). En relación a los niveles de inserción laboral de los egresados se destaca el hecho de que los egresados de enseñanza media que han cursado estudios postsecundarios presentan un mejor nivel de ocupación, alcanzando un $66.76 \%$. En cambio, las tasas de ocupación que logran los egresados de enseñanza técnico-profesional con un $62.32 \%$ y finalmente el nivel de ocupación más bajo lo obtienen los egresados de la modalidad de enseñanza Científica-Humanista que alcanza solo el $56.11 \%$.

Como podemos observar, en este estudio la educación agregada (postsecundaria) aparece como el factor más determinante en la perspectiva de lograr una mejor inserción laboral y un trabajo de mayor calidad laboral (Arzola, 1993: 24). A modo de síntesis, el estudio permite observar que el destino educacional y laboral de los estudiantes egresados de la enseñanza media es complejo y altamente inequitativo, ya sea porque un número significativo de dichos egresados $(72.2 \%)$ no continúa estudios superiores y, por otra parte, los que se logran incorporar al mercado laboral formal y/o, lo hacen en condiciones difíciles y precarias del punto de vista de los ingresos monetarios.

En este contexto, Dávila et al. (2005), al analizar las trayectorias sociales y educativas de 8.801 estudiantes secundarios (y de sus padres) pertenecientes a doce liceos municipales de las comunas de Viña del Mar, Quillota y Puchuncaví; identifica cinco escenarios postsecundarios posibles declarados por los estudiantes frente a la pregunta acerca de sus proyectos al egresar de la educación media. Dichos escenarios son: i) encontrar un trabajo $(81,6 \%)$ ii) continuar estudios superiores $(35,3 \%)$ iii) trabajar y estudiar $(23,7 \%)$ iv) trabajar un tiempo y luego estudiar y v) no lo tengo claro (14.4\%). Cabe señalar que 
en este estudio se identificó el nivel de escolaridad de la madre como principal predictor de los proyectos post egreso de los estudiantes secundarios. Entre sus principales conclusiones, se destaca el hecho que mientras más bajo dicho nivel de escolaridad, mayor es la proporción de jóvenes que piensan en no continuar estudios superiores y viceversa.

Por otra parte, Corica (2012) indaga en las expectativas sobre el futuro educativo y laboral en el marco de la escuela secundaria argentina, se realiza un breve recuento de las investigaciones sobre las expectativas de futuro y representaciones de los jóvenes en relación a la educación y al trabajo. En este estudio, pone en evidencia la diversidad de experiencias escolares de los jóvenes de distintos grupos sociales y la profunda segmentación de las oportunidades de inserción social post-secundaria. En el mismo estudio, se destaca que en el discurso de los estudiantes el paso por la educación secundaria ya no garantiza la movilidad social ascendente o una favorable inserción social. Estos hallazgos coinciden con lo que señalan diversos autores (Filmus, Kaplan, Miranda y Moragues, 2001) en relación a que la educación (primaria y secundaria) no garantiza una mejor inserción laboral, aún cuando efectivamente sea una simple condición (subjetiva) necesaria para lograr dichos objetivos sociales.

Como podemos observar las diversas políticas educativas implementadas en Chile y Latinoamérica, han generado una amplia cobertura de la educación secundaria, en co-existencia con un sistema educativo segregado y altos grados de inequidad. En este contexto, es posible sostener que las expectativas de futuro estarían moduladas por dichos factores estructurales. No obstante ello, en este estudio se plantea la necesidad de profundizar en el modo en que los estudiantes explicitan el sentido de futuro desde su experiencia escolar. Ello tiene relevancia, pues los estudiantes al tomar distancia crítica de las múltiples expresiones de inequidad y de los bajos niveles de calidad de la educación secundaria incorporan estas percepciones en sus propios proyectos de futuro.

\section{CALIDAD, EQUIDAD Y SENTIDO DE FUTURO: APROXIMACIÓN DESDE EL DISCURSO DE LOS ESTUDIANTES SECUNDARIOS}

El foco de este artículo está puesto en explorar las expectativas de futuro de los estudiantes que asisten a liceos municipales en Chile y de qué modo este sentido de futuro nos plantea diversas interrogantes y paradojas en torno a la calidad y equidad del sistema educativo. De este modo, se profundiza en esta noción en los discursos sobre educación y los procesos de escolarización secundaria. Aproximarnos a dichas paradojas desde la experiencia escolar de estudiantes de liceos municipales, nos permite comprender aspectos clave de la escolarización secundaria: ¿Cuáles son los aportes de este nivel educativos a las trayectorias sociales de los estudiantes? ¿Cómo evalúan el aporte de la "educación media municipal" en sus proyectos de futuro? Entre otras preguntas de interés.

Desde esta perspectiva, la noción de futuro es un tópico que permitirá dar cuenta tanto de factores sociales como subjetivos implicados en los procesos de implementación y mejoramiento de la escolarización secundaria. Lo anterior es reafirmado por Corica (2012), quien sostiene que no sólo los cambios ocurridos en el mercado del trabajo y en el sistema educativo operan en la visión de futuro de los jóvenes; sino, adicionalmente, existen otros factores sociales y representacionales que inciden en la configuración de sus representaciones sobre el futuro, al respecto menciona: a) lo económico-social b) 
lo individual y subjetivo c) la segmentación social y escolar d) aspectos geográficos e) género. Al respecto, los estudiantes, al narrar sus expectativas de futuro, tienden a destacar las diferencias sociales y económicas y que los jóvenes de bajos recursos no tendrían las mismas oportunidades que aquellos pertenecientes a los sectores medios y altos. Es decir, los que tienen más posibilidades de progresar son los que están en mejor posición económica. A la inversa, los jóvenes de menores recursos tienen una inserción temprana en ocupaciones precarias, inestables y poco calificadas. En estos mismos relatos, surge que <ser hijo de>, <tener tal apellido>, <vivir en tal o cual barrio $>$ genera situaciones de desigualdad que no tienen que ver con una desigualdad en el plano del conocimiento sino con diferencias legitimadas en un contexto más amplio de desigualdad social.

\subsection{ENCUADRE METODOLÓGICO DE LA INVESTIGACIÓN}

\subsubsection{Enfoque de investigación}

En el presente estudio ha optado por un enfoque metodológico cualitativo, considerando que uno de los objetivos básicos es conocer y describir la noción de futuro desde la experiencia escolar de los estudiantes que asisten a liceos municipales. Desde este paradigma, interesa conocer los procesos subjetivos de los actores educativos. Lo anterior supone adentrarse en su experiencia bajo una lógica comprensiva y guiado por preguntas que permitan develar las significaciones que subyacen a su experiencia escolar.

\subsubsection{Recolección y análisis de datos}

Uno de los rasgos distintivos de los diseños cualitativos (Flick, 2004) es que los materiales para el análisis se expresan a través del lenguaje y procesos comunicativos. En nuestra investigación se optó por utilizar la entrevista en profundidad como principal técnica de acceso al campo de estudio. Esta técnica aportó un valioso material para su posterior análisis. Para ello, se aplicó un modelo lingüístico de orientación semántica (Greimas, 1971, Martinic, 1992, Piret et al., 1996), lo cual permitió la identificación de estructuras de significado sobre las cuales se articula globalmente el discurso de los sujetos sobre el futuro postsecundario. Esta metodología de análisis permite indagar sobre las configuraciones de sentido de futuro y subjetividades de los estudiantes secundarios entrevistados.

\subsubsection{Universo y participantes del estudio}

En términos operativos, los sujetos del estudio corresponden a 32 estudiantes de cuarto año de educación media pertenecientes a cuatro establecimientos de educación pública en diferentes comunas de la Región Metropolitana (Santiago, Chile). Mediante la realización de entrevistas en profundidad a cada uno de los estudiantes se obtuvo un corpus de datos que forma parte del material que se analiza en el presente estudio.

\subsection{PRESENTACIÓN Y DISCUSIÓN DE RESULTADOS}

La presentación y análisis de los datos se realiza en torno a dos ejes semánticos globales que expresan aspectos claves del estudio: a) Sentido de futuro, el cual agrupa 
un conjunto de estructuras semánticas que emergen en los discursos de los estudiantes y que dan cuenta de una radical oposición entre presente y futuro. b) Valorización de la educación secundaria municipal. Este segundo eje semántico está constituido por un conjunto de oposiciones semánticas que remiten a juicios evaluativos respecto de la calidad del proceso formativo. Ambos ejes semánticos emergen en el discurso de los estudiantes y son analizados siguiendo los procedimientos metodológicos de la semántica estructural.

\subsubsection{Sentido de Futuro: oposición entre presente y futuro.}

El discurso de los estudiantes sobre la dimensión "temporal" se organiza en torno a un tópico central que alude a la distinción entre presente y futuro. De este modo, el discurso de los estudiantes sobre el sentido de futuro de la educación media municipal se estructura en torno a un eje semántico temporal que se expresa en las en las siguientes estructuras semánticas paralelas:

\begin{tabular}{|c|c|}
\hline \multicolumn{2}{|c|}{ TEMPORALIDAD DEL } \\
SENTIDO DE FUTURO \\
\hline Presente & Futuro \\
\hline Ahora & Después \\
\hline
\end{tabular}

Como podemos observar, sobre este eje temporal surgen las siguientes disyunciones: presente/futuro; ahora/después; las cuales sirven de elemento articulador y soporte desde el cual los estudiantes elaboran su sentido de futuro. En la relevancia asignada al presente, se destacan las experiencias, aportes y valoraciones actualizadas en el "aquí y ahora" de una temporalidad presente que da sentido a la experiencia escolar y que, en consecuencia, no necesariamente se proyecta hacia un tiempo/escenario futuro (de mediano o largo plazo). De este modo, para los estudiantes la educación secundaria adquiere un valor en el momento presente (Ahora): "por eso yo digo que hay que disfrutar la enseñanza media en este momento, las cosas que pueda ver, que pueda tocar, sentir de mi enseñanza, recibirlas con alegría y eso es lo que yo siento de la enseñanza media" (E6, LP, L, Hombre, p.14). En esta afirmación se observa que para los estudiantes la educación secundaria tiene un valor por sí misma (y tal vez per se) en el contexto global de su ciclo de vida. Esta valoración positiva es compartida por los estudiantes de todos los establecimientos

Sin embargo, a la hora de responder a la pregunta por el sentido más global de la enseñanza media, los estudiantes se posicionan, claramente, en uno u otro polo de la disyunción temporal: presente/futuro. Lo que genera claramente dos tendencias en la configuración de sentidos de la educación media municipal y las expectativas de futuro. Por una parte, se observa una tendencia "presentista" que indica que la enseñanza media tiene un/os sentido(s) que no se construye necesariamente en función de un escenario posterior (futuro). Y, por otra parte, una segunda tendencia que podemos caracterizar como "futurista", es decir, los estudiantes enfatizan la noción de futuro haciendo referencia 
directa hacia una dimensión del sentido que se proyecta hacia un tiempo desconocido e incierto. Esta visión de un futuro que otorga sentido a la enseñanza secundaria proyectado hacia un momento posterior, es predominante en el discurso de los estudiantes de liceos municipales de estrato socioeconómico medio y medio-alto.

Como hemos observado anteriormente, las estructuras semánticas que emergen reiterativamente en los textos analizados, dan cuenta de una radical oposición entre presente y futuro. Por su parte, los estudiantes de estratos socioeconómicos bajos profundizan el sentido de la educación secundaria en un "presente", mientras que en los estudiantes de estratos medios y altos se orientan literalmente hacia un "futuro" concebido como inserción social postsecundaria. Esta oposición evidencia el lugar preponderante que ocupa para los estudiantes el liceo municipal, pues mientras unos sobrevaloran este espacio (lugar seguro, protegido, para pasarlo bien); para otros constituye sólo una etapa de riesgos e incertidumbres, pero necesaria para proyectarse hacia un futuro profesional o laboral que les permita alcanzar diversas metas personales y familiares. Esta disyunción (presente/ futuro) se constituye en una dimensión de temporalidad social desde la cual se construyen los sentidos de futuro de la educación media municipal. Desde esta perspectiva, tal como señala Elias (2010), el tiempo se presenta entonces como una dimensión relativa, subjetiva y convencional cuya medida está directamente ligada a las actividades primordiales de la sociedad. Esta representación de la temporalidad social, a nuestro juicio, es una potente clave analítica que permite comprender el proceso de construcción del sentido de futuro que realizan los estudiantes secundarios en el Chile contemporáneo.

\subsubsection{Valoraciones sobre la calidad de la educación media municipal}

La valoración de los estudiantes en torno a la calidad de la educación media se manifiesta de diversas maneras en el discurso de los estudiantes. En el análisis de los datos emergen los siguientes tópicos: a) conocimientos y funcionalidad de la educación, b) valorización de la calidad de las clases (contenido, dinámica y niveles de exigencia), c) valoración de los profesores del liceo municipal y d) valoración de la disciplina y la convivencia escolar.

4.2.2.1. Valorización de los conocimientos adquiridos en la enseñanza media

Uno de los aspectos claves de las valoraciones que realizan los estudiantes en torno a la enseñanza media se relaciona con los conocimientos y los aprendizajes adquiridos a lo largo de su experiencia escolar. Esta valoración es la que presenta una mayor variabilidad de acuerdo a los distintos estratos socioeconómicos en los cuales se localizan los liceos municipales y por ello hemos escogido algunos textos pertenecientes a estudiantes de distintos liceos municipales. En el proceso de valorización de los conocimientos adquiridos en la enseñanza media municipal un conjunto de expresiones se refieren directamente a la calidad y reconocimiento de los conocimientos adquiridos a lo largo de su experiencia escolar. Al respecto la estructura paralela construida es la siguiente:

\begin{tabular}{|c|c|}
\hline \multicolumn{2}{|c|}{ CONOCIMIENTOS (TC) } \\
\hline Básicos $(-)$ & $($ Avanzados) $(+)$ \\
\hline Elementales $(-)$ & Complejos $(+)$ \\
\hline
\end{tabular}




\begin{tabular}{|c|c|}
\hline Simples (-) & (difíciles) $(+)$ \\
\hline Superficiales) (-) & Profundos (+) \\
\hline
\end{tabular}

Por otra parte, en el análisis de los planteamientos de los estudiantes surge un segundo elemento de valorización que está relacionado directamente con la calidad de los conocimientos adquiridos en la enseñanza media municipal. Este tópico de evaluación está referido a la funcionalidad (potencial) de dichos conocimientos en los diversos escenarios (actuales y futuros) en los cuales ellos mismos se ubican al momento de hablar de estos temas. Por ello, luego de un análisis semántico estructural de los discursos surge la siguiente estructura paralela:

\begin{tabular}{|c|c|}
\hline \multicolumn{2}{|c|}{ FUNCIONALIDAD } \\
\hline Igual sirven & (No sirven) \\
\hline Usar & (No usar) \\
\hline Útiles & (Inútiles) \\
\hline
\end{tabular}

Como se puede observar, los estudiantes de los liceos municipales de estratos Bajo y Medio -Bajo valoran los conocimientos recibidos en la enseñanza media municipal, sin embargo, los hacen apelando a un elemento condicional: "Uno no sabe si los va a usar o no, si sirven o no, eso se va a saber después cuando estemos afuera, es decir, si le dan continuidad en la educación superior, o en el mundo del trabajo". No obstante, el tener conciencia de la baja calidad de dichos conocimientos los hace poner una nota de escepticismo a esta funcionalidad potencial. Es destacable el hecho de que los aprendizajes en el ámbito de competencias genéricas (o transversales) sean valorados positivamente, pues reconocen que les han permitido "crecer como persona", "aprender a comunicarse e interactuar con los adultos", "estar informados", entre otros significados destacados por los estudiantes.

\subsubsection{Valorización de las clases (contenido, dinámica y niveles de exigencia)}

Desde la experiencia de los estudiantes, la valoración (positiva o negativa) sobre el contenido de las clases se construye desde un triple punto de vista: a) valoración del contenido curricular, b) valoración de su dinámica pedagógica-relacional y c) valoración de los niveles de exigencia académica (por parte de los profesores), que se verifican al interior de la sala de clases y en relación al trabajo escolar. Estos tópicos de valoración de la enseñanza media se vinculan directamente con los niveles de calidad percibidos por parte de los estudiantes que asisten a los liceos municipales. 
A continuación presentamos textos seleccionados para el análisis del eje semántico -calidad de las clases-. A partir ellos, hemos construido la siguiente estructura semántica paralela que da cuenta en forma sintética de las principales valoraciones respecto a las clases (o de las prácticas pedagógicas):

\begin{tabular}{|c|c|}
\hline \multicolumn{2}{|c|}{ Calidad de las clases } \\
\hline Buena (+) & Mala (-) \\
\hline Completas (+) & (Incompletas) \\
\hline Normales $(+)$ & Anormales (-) \\
\hline Preparada (+) & (No preparada) \\
\hline
\end{tabular}

Como podemos observar, en las estructuras paralelas presentadas, los términos utilizados para calificar la calidad de las clases se organizan en torno a la disyunción: Buena/ Mala. Esta oposición indica que entre los estudiantes existe una evaluación negativa de ellas, lo que sintetiza en los siguientes significados compartidos por los estudiantes en el espacio escolar municipal: 'no se pasa toda la materia', 'no se prepara', 'los estudiantes no se motivan' y 'hay clases que son incompletas'. Cuando se les pregunta por los factores que permitirían comprender esa negativa evaluación de la "calidad de las clases", emergen factores vinculados con las insuficiencias en "lo pedagógico", "lo curricular", "lo didáctico" y "gestión educativa", entre otras dimensiones problemáticas. A modo de ejemplo, se menciona la ausencia de condiciones de infraestructura, recursos didácticos e inexistencia de materiales necesarios para realizar adecuadamente los procesos formativos. Por otra parte, a pesar de todas restricciones a la calidad, los estudiantes reconocen que existen profesores que "se las arreglan para hacer clases de buena calidad", pero se trata de excepciones que no logran institucionalizar esas "buenas prácticas pedagógicas" en el liceo municipal.

En los textos analizados, emerge el factor (in)disciplina como un obstáculo directo y permanente que impide realizar clases de buena calidad en el liceo municipal. Al respecto, surge una posible "solución" para superar dicha tensión esencial al interior de la sala de clases ("pasar materia" o "mantener la disciplina"). Desde el discurso de los estudiantes, la mejor estrategia para abordar este problema consistiría en "apoyarse en los propios estudiantes", para luego generar nuevas formas de control social sobre sus pares. De este modo, se lograría mejorar los grados de motivación, atención y una buena convivencia en el aula. Desde otro ángulo, los profesores son considerados como actores claves en pos del logro de mayores niveles de calidad. En lo sustantivo, los alumnos plantean lo siguiente: el profesor debe crear un ambiente académico adecuado al interior del aula generando normas y disciplina escolar que facilite el trabajo escolar de todos, no debe renunciar a ejercer su rol de profesor hacia el conjunto de la clase; debe incorporar a todos los estudiantes y sobre todo no dirigir su acción pedagógica sólo a un segmento de los estudiantes ("los que se sientan adelante" o "los que quieren aprender").

Por su parte, el análisis de la dinámica pedagógica de las clases es el segundo foco de atención para aproximarnos a las valorizaciones que construyen los alumnos respecto 
de este elemento clave en la construcción del sentido de la enseñanza media municipal. En este contexto es que presentamos la siguiente estructura semántica paralela:

\begin{tabular}{|c|c|}
\hline \multicolumn{2}{|c|}{ DINÁMICA PEDAGÓGICA } \\
DE LAS CLASES (TC) \\
\hline Lentas (-) & (Rápidas) $(+)$ \\
\hline Aburridas (-) & (Entretenidas) $(+)$ \\
\hline No interesantes (-) & (Interesantes) $(+)$ \\
\hline No motivadoras $(-)$ & (Motivadoras) $(+)$ \\
\hline
\end{tabular}

El último foco de análisis del eje 'calidad de las clases' está referido a determinar las actitudes que los estudiantes tienen respecto de los niveles de exigencia que se observan tanto en las clases como en las actividades asociadas a ellas en el marco de la rutina de trabajo pedagógico al interior del aula. A partir de estos textos surge un eje semántico que da cuenta de los niveles de exigencia percibidos por los estudiantes y que se grafica de la siguiente forma:

\begin{tabular}{|c|c|}
\hline \multicolumn{2}{|c|}{ NIVEL DE EXIGENCIA } \\
\hline Baja & $($ Alta $)$ \\
\hline Simplificar $(-)$ & (Complejizar) $(+)$ \\
\hline Fácil $(-)$ & (Difícil) $(+)$ \\
\hline Mínimo & (Máximo) \\
\hline
\end{tabular}

Como se puede observar en el esquema anterior, los lexemas identificados se organizan en torno al eje semántico del nivel de exigencia, el cual es visto preferentemente desde la perspectiva de la exigencia (unilateral) que hace el profesor a los alumnos, respecto a los grados de dificultad que se le imprime a una determinada materia o contenido. Desde otro punto de vista, el nivel de exigencia interpela a los propios alumnos a desarrollar ciertas conductas y estrategia de trabajo escolar, es decir, la exigencia se construye en torno a un acuerdo tácito entre ambos actores escolares.

\subsubsection{Valorización de los profesores}

La valorización que hacen los estudiantes entrevistados respectos de los profesores abarca dos dimensiones claramente diferenciadas: valorización afectiva y una valoración racional de la acción docente. En el primer tópico, emergen significados relacionados con los valores del profesor, su trato con los alumnos, su capacidad de escucha y empatía. En el segundo tópico, se valorizan sus conocimientos, 'su pedagogía', es decir, su calidad profesional. De ambas valorizaciones se nutre el proceso de construcción de sentido sobre la enseñanza media. Sobre la base de considerar ambas dimensiones de análisis antes indicadas y siguiendo los discursos de los estudiantes, en los cuales se identifican diversas fuentes de valorización tanto positiva como negativa de los profesores, se han elaborado las siguientes estructuras semánticas paralelas: 


\begin{tabular}{|c|c|}
\hline \multicolumn{2}{|c|}{ COMPROMISO (TC) } \\
\hline Presencia $(+)$ & (Ausencia) $(-)$ \\
\hline Preocupados $(+)$ & (Despreocupados) $(-)$ \\
\hline Ayudan $(+)$ & (No ayudan) $(-)$ \\
\hline Aconsejan $(+)$ & (No aconsejan $(-)$ \\
\hline Motivan $(+)$ & (Desmotivan) $(-)$ \\
\hline
\end{tabular}

Como podemos observar, la principal fuente de valorización positiva de los profesores en la enseñanza media municipal se estructura en torno a la oposición profesor presente / profesor ausente. En la mayor parte de los textos analizados se destaca la presencia permanente de los profesores de la enseñanza media municipal, que se expresa fundamentalmente en una serie de acciones que realizan y que denotan preocupación, principalmente frente a los diversos problemas que afectan a los alumnos, los que generalmente van más allá de su rendimiento estrictamente académico.

Cabe señalar que dicha preocupación involucra diversas dimensiones de la experiencia social y escolar de los alumnos; como, por ejemplo, problemas de identidad, situaciones familiares críticas, conflictos de relación interpersonal entre los mismos alumnos y, finalmente, se destaca una permanente preocupación por el rendimiento académico de cada uno de los alumnos.

En cambio, la valoración negativa de los profesores que se desempeñan en la enseñanza media se estructura en torno a una serie de elementos como las prácticas docentes deficientes, los niveles de exigencias académicas bajos, problemas asociados al ausentismo laboral y a la organización del tiempo del trabajo escolar al interior del aula. De todos modos, se valora la preocupación constante de los profesores por la 'parte humana de los alumnos', es decir, por sus problemas personales y familiares. En prácticamente todos los textos recopilados, la valoración positiva de los profesores se articula fundamentalmente en torno a una serie de elementos como su buena disposición a ayudar, su apertura a los problemas personales y familiares del alumno, su capacidad para realizar su docencia en condiciones de desinterés y desmotivación por parte de los alumnos, lo que en ocasiones hace difícil la tarea de desarrollar las clases normalmente.

\subsubsection{Valorización de la disciplina y las normas en la enseñanza media municipal}

Para realizar una delimitación de las actitudes específicas que tienen los estudiantes sobre la disciplina y las normas al interior del liceo, hemos seleccionado algunos de los textos más representativos para cada uno de los casos. A partir de los textos analizados (ver anexo) hemos construido las siguientes estructuras paralelas: 


\begin{tabular}{|c|c|c|c|}
\hline \multicolumn{4}{|c|}{ (TC) DISCIPLINA } \\
\hline \multicolumn{2}{|c|}{ Buena } & \multicolumn{2}{|c|}{ (Mala) } \\
\hline \multicolumn{2}{|c|}{ Control } & \multicolumn{2}{|c|}{ (Descontrol) } \\
\hline \multicolumn{2}{|c|}{ Desorden focalizado } & \multicolumn{2}{|c|}{ Desorden (generalizado) } \\
\hline \multicolumn{2}{|c|}{ Liceo no paqueado } & \multicolumn{2}{|c|}{ (Liceo Paqueado) } \\
\hline \multirow[t]{2}{*}{ Control bajo } & \multirow[t]{2}{*}{ (Sin controles) } & (Control Excesivo) & (Fuera de Control) \\
\hline & & (Vigilancia efectiva) & (Vigilancia al cuete) \\
\hline
\end{tabular}

En las estructuras semánticas que describen el eje semántico disciplina, esta se organiza en torno a la oposición: buena/mala; control/descontrol. Estos términos indican que en los liceos municipales estudiados existe una valoración negativa de la disciplina. Los estudiantes señalan que existe un sistemático desbordamiento de las normas disciplinarias tanto al interior del aula como al interior del propio liceo. Por ello, el modo de producir disciplina escolar es el permanente control, la supervisión por parte de los inspectores en el patio e, incluso, en casos extremos, la inspección al interior mismo de las propias aulas de y durante el desarrollo de las clases. Es un hecho común que en todos los liceos los estudiantes declaran la presencia de un desorden sistemático y focalizado en ciertas asignaturas, con ciertos profesores y horarios específicos de la jornada escolar.

Para referirse a esta realidad de (in)disciplina un estudiante nos relata lo siguiente: "lo malo en este liceo son los segundos planes que uno tiene, que nos escapamos a las doce pa' ir a tomar, uno está pensado en divertirse y no tanto en clase". Como se puede observar estos segundos planes son un quebramiento consciente, planificado y reiterado de la disciplina escolar. Al preguntársele al mismo estudiante acerca de cómo surgen estos, responde lo siguiente:

"del momento en que uno se siente mal, o la clase esta muy aburrida y hay jornada completa y yo encuentro que la jornada completa no ayuda en nada de nada, estamos aburridos $y$ de repente decimos por que no vamos a tomar hoy día, o vamos pa' allá, o vamos a ver el partido que es a las doce y no importa, nos escapamos no mas total la profe no se da cuenta" (E8, LP, Daniel, p.10).

Estos segundos planes se pueden realizar en los liceos donde hay una relativa libertad por parte de los estudiantes, siendo los mecanismos de control poco eficientes y sostenidos en la labor disciplinaria que ejercen los propios profesores al interior del aula y/o de los inspectores en los horarios de esparcimiento al interior de los establecimientos.

A partir de las estructuras semánticas anteriormente identificadas, surge una estructura subordinada que se articula sobre la oposición liceo paqueado/ liceo no paqueado. El primer término indica que se trata de un tipo de disciplina conseguida a partir de un control tal vez excesivo y permanente en todos los espacios del liceo. Esta disciplina se ejerce en forma coactiva y se extiende durante toda la jornada escolar.

En el caso de los liceos donde se ejerce este tipo de disciplina, los estudiantes definen a su propio liceo como liceo paqueado en alusión a la disciplina que se impone con los Carabineros o pacos. A pesar de este nivel de vigilancia en el caso de algunos liceos, la disciplina es quebrantada permanentemente e impide realizar normalmente las actividades académicas. Como podemos observar, al interior de los liceos se verifica una permanente 
tensión entre una tendencia al orden y al respeto a las normas de funcionamiento escolar y por otra parte en los liceos existen diversos mecanismos de quebrantamiento de dicho orden. Lo anterior refleja una clara lucha de poder entre grupos del alumnado y por otra parte, las distintas autoridades al interior de la institución escolar (Directores, inspectores, etc.). Esta tensión es resuelta en ocasiones en "favor de los estudiantes" y se expresa en una indisciplina generalizada y la mayor parte de las situaciones se resuelve con más control, supervisión y sanciones que buscan restablecer el orden escolar.

En los textos analizados se observa que en la base de las valorizaciones que realizan los estudiantes sobre la disciplina al interior de los liceos municipales, se encuentra un juicio crítico sobre la (in)capacidad integradora, es decir, hay discursos que revelan la existencia de liceos con una organización interna potente que es capaz de integrar a todos los estudiantes en el marco de su misión formativa. Por otra parte, los textos nos hablan de liceos que no logran constituirse en un espacio integrador que promueva a todos los estudiantes en el logro de sus metas en la vida, lo que se plantea en los siguientes términos:

"Igual tengo ese remordimiento [pena] de no haber estado en otro colegio, igual yo cacho que uno hace la diferencia y yo trato de (II) hacer la diferencia, pero aquí nadie pesca, es puro desorden, puro leseo, es como que todos se dedican a puro vivir el momento en la sala [de clases], cero responsabilidad, cero estudio, salvo contadas ocasiones, y con ciertos profes, y es como que sólo en algunos momentos esto se transforma en una verdadera escuela" (E1, SR, Cinthya, p.24).

En este nivel de análisis, se puede afirmar que los jóvenes tienden a internalizar una valorización de la disciplina como reglas del juego mínimas que hacen (im)posible una convivencia escolar en pos de los aprendizajes de calidad. A modo de conclusión preliminar, podemos afirmar que el atributo de la disciplina en el aula es altamente valorado desde la subjetividad de los jóvenes estudiantes que asisten a liceos municipales y, más aún, en esa capacidad de "imponer disciplina" por parte del profesor se juega una parte significativa de la actitud o valoración global sobre la calidad del liceo municipal.

\section{CONCLUSIONES}

Una primera afirmación de este trabajo se relaciona con la centralidad que adquiere la dimensión temporal en los discursos de los jóvenes estudiantes secundarios entrevistados; es decir, cuando estos se refieren a su experiencia escolar, lo hacen apelando directamente a sus aspiraciones en la vida, sus necesidades y sueños futuros. Y lo hacen situándose en un contexto social marcado por las condiciones de precariedad, vulnerabilidad social e incertidumbre hacia el futuro. Al respecto, diversos autores (Lechner 2003; Elias, 2010 Corica, 2012) han señalado que la dimensión temporal ocupa un lugar central en las sociedades contemporáneas, las cuales se caracterizan por una un cierto repliegue temporal de los sujetos; lo cual significa que vivimos en torno al presente como tiempo único, que se caracteriza por la simultaneidad, su velocidad y la transmisión simultánea de imágenes virtuales. De este modo, se plantea que, en el tiempo histórico, se entrelazan experiencias cotidianas con horizontes de futuro en el largo plazo. Estos horizontes temporales no son proyecciones de metas ni planes a cumplir, más bien son "constructos" o apuestas acerca del sentido que atribuimos al trayecto realizado [o a una parte de él] y a las expectativas de un futuro mejor. Visto así, hacer políticas educativas consiste en 
producir los horizontes de sentido que permiten a los actores sociales poner sus vivencias y evaluaciones en perspectiva y específicamente en el ámbito del diseño de políticas de mejoramiento de los procesos de escolarización secundaria.

Un segundo aspecto que surge de la investigación muestra que los estudiantes, junto con evaluar la calidad en educación media municipal, paradojalmente, tienden a valorizar negativamente aspectos clave de la calidad de la educación municipal. Sin embargo, simultáneamente evalúan positivamente su funcionalidad global en pos de una futura inserción laboral y/o continuidad de estudios superiores. Para ellos, a pesar de los bajos niveles de calidad exhibidos por la educación media municipal; igual sirve; igual algo se aprende $\mathrm{y}$, tal como dice un estudiantes: "Uno no sabe (...) si sirven o no, eso se va a saber después cuando estemos afuera, es decir, si le dan continuidad en la educación superior, o en el 'mundo del trabajo'." Desde esta perspectiva, el tener conciencia de la baja calidad de dichos conocimientos los hace poner una nota de incertidumbre a la funcionalidad futura de la educación municipal. En todo caso, desde su experiencia actual, los conocimientos que más aportan a su formación no son de orden académicos sino más bien aquellos que les han permitido "crecer como persona", "aprender a comunicarse e interactuar con los adultos", "estar informados" y desarrollar la sociabilidad, la capacidad de conversar con otros, "tener más roce social", "tener más cultura" y "aprender a conversar".

Al respecto, Makuc (2009), al analizar la noción de temporalidad entre estudiantes de un liceo público en la zona sur de Santiago, señala que las certezas e incertidumbres forman parte de las conversaciones de los jóvenes en relación al significado de futuro. Cuando los estudiantes hablan de las certezas del presente no emergen inmediatamente los déficit en sus condiciones materiales de vida (recursos económicos o sociales); sino que estas deficiencias sólo se expresan en el discurso cuando se refieren a sus proyectos individuales para el futuro. De este modo, los bajos niveles de calidad de la educación secundaria que reciben son un obstáculo para estructurar dichos proyectos y en consecuencia limitan su capacidad de agencia. Dichas incertidumbres, actuarían a modo de pruebas (sociológicas) que se expresan en pobreza, carencias afectivas y materiales, droga, delincuencia; las cuales desafían cotidianamente al sujeto a distanciarse reflexivamente y lograr constituirse en actor de su propia experiencia escolar (y social).

Entre las conclusiones principales interesa destacar la paradoja de equidad que emerge en el discurso de los estudiantes y que específicamente surge cuando ellos perciben que, al egresar de la educación media con una baja formación, no es posible ingresar al mundo del trabajo y menos aún continuar estudios superiores. En cambio, sostienen que los estudiantes de otros liceos de mejor calidad (algunos particulares subvencionados con co-pago y particulares pagados) pueden optar sin mayores dificultades a estudiar en la educación superior. En este sentido, resultaría pertinente en futuras estrategias de intervención el fortalecimiento de las concepciones de la temporalidad social que configuran los estudiantes secundarios y, concretamente, fortalecer sus capacidades en el manejo/ gestión del tiempo escolar, en pos de sus propios objetivos, sus proyecciones de futuro y las posibilidades de superar los factores que los excluyen de las posibilidades de desarrollo personal y social que ofrecen las políticas públicas y educativas.

Por otra parte, es necesario destacar que en el discurso de los estudiantes el paso por educación secundaria ya no garantiza la movilidad social ascendente o la inserción social de los egresados. Lo anterior, es coincidente con lo que señalan diversos autores (Filmus, Kaplan, Miranda y Moragues, 2001): que la educación primaria y secundaria 
ya no garantiza una mejor inserción laboral, aun cuando efectivamente sea una simple condición (subjetiva) necesaria para lograr dichos objetivos sociales.

Finalmente, resulta relevante destacar la existencia de pluralidad de sentidos que emergen desde la experiencia escolar de los estudiantes secundarios que asisten a la educación media en Chile. Igualmente, nos parece pertinente resaltar que en dichas configuraciones de sentido se expresa una paradoja central identificada en el discurso de los estudiantes: si la escuela secundaria tiene como principal sentido el prepararse para el futuro, ¿en qué medida los actuales niveles calidad medidos por diversos instrumentos y declarados por los estudiantes en su propia experiencia escolar, permiten responder a esta expectativa de futuro académico y laboral destacado por los jóvenes estudiantes que asisten a las escuelas secundarias públicas en Chile? En la perspectiva de superar esta paradoja, la educación pública tiene en la actualidad entre sus principales desafíos la tarea de (re) construir el sentido democrático y de integración social de la educación pública chilena.

\section{REFERENCIAS BIBLIOGRÁFICAS}

Araujo, K. \& Martucelli, D. (2012). Desafíos comunes. Retrato de la sociedad chilena y sus individuos. Tomo I. Neoliberalismo, democratización y lazo social. Santiago: LOM.

Arzola, S.; Collarte, C.; Cornejo, J.; Etchegaray, F. Franklin, J. (1993). Destino Educativo Laboral de los Egresados de Enseñanza Media. Informe de Estudio de Seguimiento Proyecto Mece Media. Santiago: Ministerio de Educación.

Bellei, C. (1995). Los debates sobre educación media chilena en el Siglo XX (1910-1973). En: Estudios Sociales, n. 85, t. 3, 11-35.

Bellei, C. \& Gonzalez,P. (2010). Educación y Competitividad en Chile. En línea, disponible en www.educarchile.cl

Bellei, C. (2012) Políticas educativas para el nivel secundario: complejidades y convergencias. En E. Tenti (coord.), La escolarización de los adolescentes: desafíos culturales, pedagógicos y de política educativa. Buenos Aires: UNESCO/Instituto Latinoamericano de Planeamiento Educativo/ Instituto Latinoamericano de Planeamiento Educativo.

CEPAL (2000). Panorama Social de América Latina 1999-2000. Santiago : Comisión Económica para América Latina y el Caribe.

CEPAL (2008) Juventud y cohesión social en iberoamérica. Un modelo para armar. Santiago: Comisión Económica para América Latina y el Caribe.

CEPAL (2011). Panorama Social de América Latina 2011. Santiago : Comisión Económica para América Latina y el Caribe.

Cruz, N. (2002). El surgimiento de la educación secundaria pública en Chile. 1843-1876. (El plan de Estudios Humanistas). Santiago: DIBAM/PIIE/CIDBA.

Corica, A. (2012). Las expectativas sobre el futuro educativo y laboral de jóvenes de la escuela secundaria: entre lo posible y lo deseable. Última Década, $n$. 36, 71-95.

Dávila, O., Ghiardo, F., Medrano, C. (2005). Los desheredados. Trayectorias de vida y nuevas condiciones juveniles. Valparaíso: CIDPA EDICIONES.

Elias, N. (2010). Sobre el tiempo. México: Fondo de Cultura Económica.

Ferreira, H. (2006). Transformación de la educación media en la argentina. Tensiones y conflictos en el diseño e implementación en la Provincia de Córdova. Argentina: Editorial Universidad Católica de Córdova.

Flick, U. (2004). Introducción a la investigación cualitativa. Madrid: Morata.

Filmus, D., Kaplan, C., Miranda, A., Moragues, M. (2001). Cada vez más necesaria, cada vez más insuficiente, la escuela media en época de globalización. Buenos aires: Santillana. 
Flecha, R. et al. (2004). Retos y salidas educativas en la entrada del siglo. En F. Imbernón (coord.), La educación en el siglo XXI. Los retos del futuro inmediato. España: Grao.

García- Huidobro, J. E. (2010). Para Hacer pública la educación pública. En línea; disponible en: http://www.cide.cl

Greimas, A.J. (1971). Semántica estructural. Investigación metodológica. Madrid: Gredos

Lechner, N. (2003) ¿Cómo reconstruimos un nosotros? Boletín Revista Latinoamericana de Desarrollo Humano, n. 4, 1-17.

Makuc, I. (2009). Significación de futuro: una aproximación desde el discurso de estudiantes secundarios de la comuna de San Ramón. Tesis para optar al Grado de Magíster en Antropología y Desarrollo. Santiago: Universidad de Chile.

Martinic, S. (1992). Análisis estructural: presentación de un método para el estudio de lógicas culturales. Santiago: Centro de Investigación Desarrollo de la Educación.

Martins, J. (1997). A exclusão social e a noa desigualdade. São Paulo: Paulus.

Martuccelli, D. \& Singly, F. (2012). Las sociologías del individuo. Santiago: LOM.

MINEDUC (2010). Calidad para Todos, Ministerio de educación, Cuenta Pública 2006-2010. Santiago: MINEDUC.

MINEDUC (2009). Indicadores de la Educación en Chile 2007-2008. Ministerio de educación, Cuenta Pública 2006-2010. Santiago: MINEDUC.

OCDE (2009). Los docentes son importantes: atraer, formar y conservar a los eficientes. En línea, disponible en http://www.oei.es

OCDE (2010). Resultados PISA 2009: resumen ejecutivo. En línea, disponible en http://www.oei.es

Tedesco, J.C., Lopez, N. (2002). Desafios a la educación secundaria en América Latina. Revista de la CEPAL, n. 76, 55-69.

SITEAL (2008). La Escuela y los adolescentes: Informe sobre las Tendencias Sociales y Educativas en América Latina 2008. Buenos Aires: Sistema de Información de Tendencias Educativas en América Latina/SITEAL/OEI/UNESCO-IIPE.

Tenti, E. (2007). La escuela y la cuestión social. Ensayos de sociología de la educación. Buenos Aires: Siglo XXI Editores.

Piret, A., Nizet, J., Bourgeois, E. (1996). L' Analyse Structurale. Une méthode d' analyse de contenu pour les sciences humaines. Bruxelles: De Boeck Université.

Pereira Leaõ, G. (2012) Rumbos de la vida, sentidos de la escuela: Diálogos juveniles sobre la educación secundaria. En E. Tenti (coord.), La escolarización de los adolescentes: desafíos culturales, pedagógicos y de política educativa. Buenos Aires: UNESCO/Instituto Latinoamericano de Planeamiento Educativo.

UNESCO (2011). Compendio Mundial de la Educación 2011. Comparación de las estadísticas de la educación en el mundo. Enfoque en la Educación Secundaria. Montreal, Canada: Instituto de Estadística de la UNESCO.

UNESCO (2012). Informe Regional de Monitoreo del Progreso Hacia Una Educación de Calidad para Todos en América Latina y el Caribe 1999-2000. Santiago: UNESCO-EPT. 